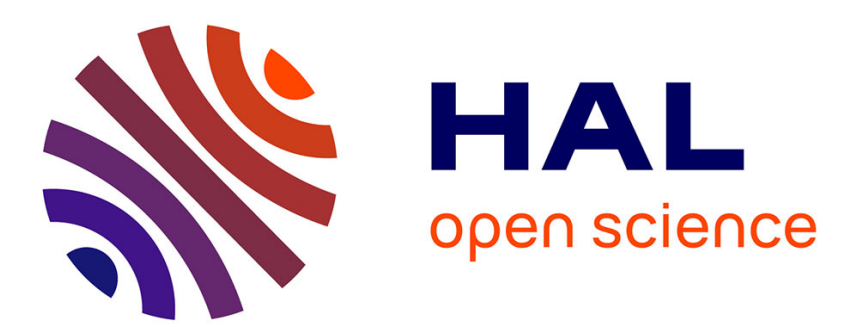

\title{
Oscillatory convective instabilities in cholesteric and smectic A liquid crystals
}

\author{
H. Pleiner, H. Brand
}

\section{To cite this version:}

H. Pleiner, H. Brand. Oscillatory convective instabilities in cholesteric and smectic A liquid crystals. Journal de Physique Lettres, 1980, 41 (16), pp.383-386. 10.1051/jphyslet:019800041016038300 . jpa00231803

\section{HAL Id: jpa-00231803 https://hal.science/jpa-00231803}

Submitted on 1 Jan 1980

HAL is a multi-disciplinary open access archive for the deposit and dissemination of scientific research documents, whether they are published or not. The documents may come from teaching and research institutions in France or abroad, or from public or private research centers.
L'archive ouverte pluridisciplinaire HAL, est destinée au dépôt et à la diffusion de documents scientifiques de niveau recherche, publiés ou non, émanant des établissements d'enseignement et de recherche français ou étrangers, des laboratoires publics ou privés. 


\title{
Oscillatory convective instabilities in cholesteric and smectic A liquid crystals
}

\author{
H. Pleiner and $H$. Brand \\ Universität Essen, Fachbereich Physik, Postfach 103764, D4300 Essen 1, R.F.A.
}

(Reçu le 28 avril 1980, accepté le 23 juin 1980)

\begin{abstract}
Résumé. - Une instabilité convective oscillante est prédite si on chauffe, par le bas, un échantillon planaire de cristal liquide cholestérique ou smectique $\mathbf{A}$.
\end{abstract}

Abstract. - We predict the occurrence of an oscillatory convective instability when a planar texture of cholesteric or smectic A liquid crystals is heated from below.

1. Introduction. - The study of convective instabilities, generated by external temperature gradients, in liquid crystals is interesting for at least two reasons. First convective instabilities are nonequilibrium phase transitions from a state of higher to a state of lower symmetry [1,2]. Since liquid crystals already have a reduced symmetry (compared to simple fluids) a further reduction of the symmetry by convective structures has to be expected and will lead to new effects. Secondly the experimental investigation of thermal instabilities in liquid crystals, which has mainly been addressed to nematics during the last years [3-6], can yield information on various hydrodynamic parameters like viscosities and static susceptibilities.

In nematics the existence of a stationary and of an oscillatory convective instability is established both theoretically [7-9] and experimentally $[3,5,6]$. The former is due to the orientational degree of freedom and to the anisotropy of the heat conduction $\kappa_{\mathrm{a}} \neq 0$.

An analogous instability based on $\kappa_{\mathrm{a}} \neq 0$ and the additional translational degree of freedom (reflecting the broken translational symmetry in one dimension) exists in cholesterics [10-12] and smectics A [12] (hereafter this instability will be called translationanisotropy instability, TAI). In the simplest approximation (e.g. temperature independent pitch or distance of the layers) the TAI is stationary $[10,11]$.

In the present letter we theoretically predict the existence of a second, manifestly oscillatory instability (OSI) in smectics A and cholesterics obtained by heating a liquid crystal planar texture from below.

2. Threshold condition. - We consider horizontal layers of smectic A or cholesteric liquid crystal between an upper and a lower plate at $z=d$ and $z=0$, respectively. The temperature difference $\Delta T$ is applied across the layers so that

$$
T(z=d)-T(z=0)=\Delta T .
$$

In the $x y$-plane the layers of smectics $\mathrm{A}$ or cholesterics are taken to be infinite, for simplicity. The preferred direction $\mathbf{p}$ of the liquid crystals is assumed to be perpendicular to the layers i.e. in our configuration parallel to the $z$-axis.

For smectics A p is identical with the averaged axis of the molecules, usually called $\mathbf{n}$, whereas for cholesterics $\mathbf{p}$ denotes the pitch axis which is perpendicular to the spiralling molecular axis $\mathbf{n}$. Furthermore we assume the presence of an external magnetic field $\mathbf{H}$ parallel to $\mathbf{p}$.

For the conserved quantities $\rho$ (mass density), g (momentum density) and $\sigma$ (entropy density) and for displacement vector of the layers $\mathbf{R}$ characterizing the broken translational symmetry along $\mathbf{p}$ we use nonlinear hydrodynamic equations whose reversible parts have been derived recently [13] and whose irreversible parts are a straightforward generalization of the linear terms given by Martin, Parodi and Pershan [14].

Although the hydrodynamic equations for smectics A and cholesterics are identical only in their linearized form to lowest order in the wave vector $\mathbf{k}$, the differences do not play a crucial role for the oscillatory instability considered here $\left({ }^{1}\right)$. Furthermore it should be noticed that the phenomenological parameters occurring in the hydrodynamic equations (viscosities and static susceptibilities) can assume different nume-

(1) The differences in the hydrodynamic equations for smectics $A$ and cholesterics are much more important for the TAI mentioned above. This point will be discussed in detail elsewhere [12]. 
rical orders of magnitude in the smectic A and cholesteric phase respectively.
The equations for smectics A read (for the notation cf. ref. [13])

$$
\begin{aligned}
& \frac{\partial}{\partial t} \rho+\nabla_{i} \rho V_{i}=0 \\
& \frac{\partial}{\partial t} \sigma+\nabla_{i} \sigma V_{i}=\nabla_{j} \kappa_{i j} C_{p} T_{0}^{-1} \nabla_{i} T \\
& \frac{\partial}{\partial t} R_{i}+V_{k} \nabla_{k} R_{i}-p_{i} p_{k} V_{k}+f_{k j} R_{i} \nabla_{k} V_{j}=\zeta \nabla_{j} \Phi_{i j} \\
& \frac{\partial}{\partial t} g_{i}+\nabla_{i} p-f_{i}^{\mathrm{ext}}+\nabla_{j} \rho V_{i} V_{j}+\nabla_{j}\left(\Phi_{k j} \nabla_{i} R_{k}-p_{i} p_{k} \Phi_{k j}-f_{j i} R_{m} \nabla_{l} \Phi_{m l}\right)=\nabla_{j} v_{i j k l} \nabla_{l} V_{k}
\end{aligned}
$$

where $p$ is the pressure, $\mathbf{f}^{\text {ext }}$ an external force density (in the present study the gravitational force $-g_{\mathrm{E}} \hat{e}_{z}$ ). The dynamical equation for $R_{i}$ contains the wellknown permeation effect.

For the linearized equations of state we have

$$
\begin{aligned}
V_{i} & =\frac{1}{\rho} g_{i} \\
\delta \sigma & =C_{p} T_{0}^{-1} \delta T \\
\delta \rho & =\chi_{\mathrm{E}} \delta p-\alpha \delta T \\
\Phi_{i j} & =p_{i} p_{j} p_{k} p_{m} \chi_{\|} \nabla_{k} R_{m}+ \\
& \quad+p_{i} p_{k}\left(\delta_{j m}-p_{j} p_{m}\right)\left|\chi_{\mathrm{a}}\right| H^{2} \nabla_{m} R_{k} .
\end{aligned}
$$

The tensor $f_{k j}$ contains two phenomenological reversible parameters $f_{1}, f_{2}$; the irreversible parameters are $\zeta$ (viscosity of the variable characterizing the broken symmetry), $v_{i j k l}$ (5 independent viscosities) and $\kappa_{\|}, \kappa_{\perp}$ (thermal conductivities); $\chi_{\mathrm{E}}, \alpha, C_{p}, \chi_{\|}$and $\chi_{\mathrm{a}}$ are static susceptibilities. In writing down equations
(1) and (2) we have neglected a dynamical (dissipative) as well as a static coupling term between $R_{i}$ and $T$ because these two terms are of no importance for the OSI considered here (for their influence on the TAI compare e.g. refs. $[11,12])$.

When the applied temperature gradients are small there exists a steady, pure heat conduction regime $(\mathbf{V}=\mathbf{0})$ with the same distribution of pressure $(p=p(z))$ and mass density $(\rho=\rho(z))$ as in simple fluids ; in addition we have $R_{i}=0$ and $\Phi_{i j}=0$ which means constant distance between the layers. The stability of the heat conduction regime is analysed by linear stability analysis within the Boussinesq approximation [15].

The resulting system of coupled algebraic equations for the frequency $\omega$ and the critical temperature difference $\Delta T$ allows for two types of solutions. One describes the TAI which is stationary $(\omega=0)$ in the approximation of equations (1) and (2) [10-12].

The second solution is connected with an oscillatory instability (OSI) with frequency

$\omega^{2}(k)=\left(\kappa_{\|} k^{4}+\hat{\mu}^{4}\right)^{-1}\left\{\zeta \hat{\chi}^{2}\left(\hat{\mu}^{4}+\hat{\kappa}^{2} k^{2}\right)\left(\kappa_{\mathrm{a}} k_{\perp}^{2}-\zeta \hat{\chi}^{2}\right)+\kappa_{\mathrm{a}} k_{\perp}^{2} \hat{\mu}^{4} \hat{\kappa}^{2}+\left(\kappa_{\|} k^{2}-\zeta \hat{\chi}^{2}\right) k_{\perp}^{2} \hat{\chi}^{2} \rho_{0}^{-1}\right\}$

and temperature difference

$$
\Delta T(k)=-\frac{\rho_{0} d}{\alpha g_{\mathrm{E}}} \frac{\hat{\mu}^{4}+\zeta \hat{\chi}^{2} k^{2}}{\hat{\mu}^{4}+\kappa_{\|} k^{4}}\left\{k_{\perp}^{-2}\left(\hat{\mu}^{4}+\hat{\kappa}^{2} k^{2}\right)\left(\zeta \hat{\chi}^{2}+\hat{\kappa}^{2}\right)+\hat{\chi}^{2} \rho_{0}^{-1}\right\}
$$

where we have used the abbreviations

$$
\begin{aligned}
\hat{\mu}^{4} & =\rho_{0}^{-1}\left(v_{3}\left[k_{\|}^{2}-k_{\perp}^{2}\right]^{2}+2\left[v_{1}+v_{2}\right] k_{\|}^{2} k_{\perp}^{2}\right) \\
\hat{\kappa}^{2} & =\kappa_{\|} k_{\|}^{2}+\kappa_{\perp} k_{\perp}^{2} \\
\kappa_{\mathrm{a}} & =\kappa_{\|}-\kappa_{\perp} \\
\hat{\chi}^{2} & =\chi_{\|} k_{\|}^{2}+\left|\chi_{\mathrm{a}}\right| H^{2} k_{\perp}^{2}
\end{aligned}
$$

$k_{\|}$and $k_{\perp}$ are the components of the wave vector $\mathbf{k}$ which are parallel and perpendicular to $\mathbf{p}$, respectively. In the geometry considered $k_{\|}^{c}$ is equal to $\pi / d$ for free-free boundary conditions ; for realistic boundary conditions one has to determine $k_{\|}^{\mathrm{c}}$ by computational means like in simple fluids [15]. $k_{\perp}^{\mathrm{c}}$ is found by mini- 
mizing $\Delta T$ with respect to $k_{\perp}^{2}$. This leads to an algebraic equation of the seventh order for $k_{\perp}^{2}$ which has to be solved for its lowest root $k_{\perp}^{\mathrm{c} 2}$ numerically. Generally one expects $k_{\perp}^{\mathrm{c}}=\sqrt{g} \frac{\pi}{d}$ where $g$ is a number of order unity.

For $\hat{\mu}^{4} k^{-2} \gg \hat{\kappa}^{2} \gg \zeta \hat{\chi}^{2}$ (i.e. viscous damping is the dominating dissipative process) $g$ is determined by

$$
\begin{aligned}
2 v_{3} \kappa_{\perp} g^{3}+\left(v_{3} \kappa_{\|}+2\left[v_{1}+v_{2}-v_{3}\right] \kappa_{\perp}+\right. \\
\left.+d^{2} \pi^{-2}\left|\chi_{\mathrm{a}}\right| H^{2}\right) g^{2}-v_{3} \kappa_{\|}=0 ;
\end{aligned}
$$

for simple fluids $g=1 / 2$.

Thus the critical temperature difference $\Delta T_{\mathrm{c}}$ and the critical frequency $\omega_{c}$ are

$$
\Delta T_{\mathrm{c}}=\Delta T\left(k_{\|}^{\mathrm{c}}, k_{\perp}^{\mathrm{c}}\right) \quad \text { and } \quad \omega_{\mathrm{c}}=\omega\left(k_{\|}^{\mathrm{c}}, k_{\perp}^{\mathrm{c}}\right) .
$$

The eigenfunctions which are associated with equation (3) allow for a pattern of two-dimensional convective rolls oscillating with frequency $\omega_{\mathrm{c}}$.

The critical temperature difference $\Delta T_{\mathrm{c}}$ in equation (3) is negative, i.e. the oscillatory instability sets in when heating from below. At the moment it is not possible for us to give numerical values for $\Delta T_{\mathrm{c}}$ for smectic A liquid crystals, because there seems to exist a lack of accurate data for some of the parameters involved. Probably the measurement of $\Delta T_{\mathrm{c}}$ will yield further information on some of the parameters. For cholesterics $\left|\Delta T^{\mathrm{c}}\right| \approx 1 \mathrm{~K}$ (for $d=1 \mathrm{~cm}$, pitch $p_{0}=50 \mu$, MBBA [10]), which is the same order of magnitude as $\Delta T^{\mathrm{c}}$ for TAI.

The critical frequency $\omega_{\mathrm{c}} \approx 5 \times 10^{-2} \mathrm{~Hz}$. Both $\omega_{\mathrm{c}}$ and $\left|\Delta T^{\mathrm{c}}\right|$ will rise, if $d$ and $p_{0}$ are lowered.

In figure 1 we have plotted schematically the

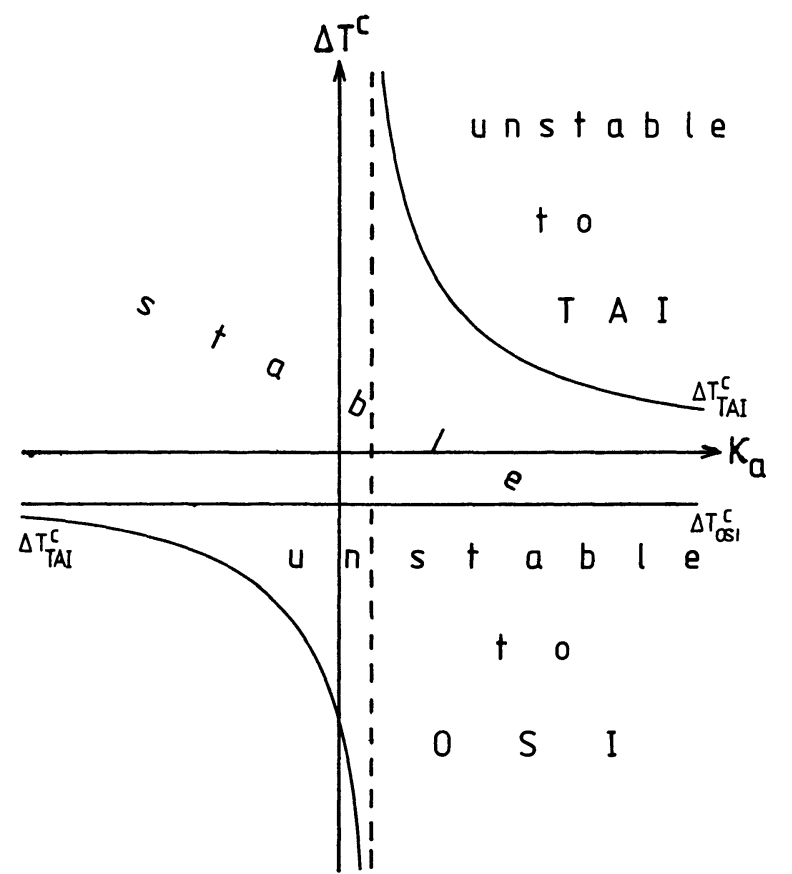

Fig. 1. - Regions of (in)stability for smectics A and cholesterics subject to vertical temperature gradients as a function of the heat diffusion anisotropy. regions of (in)stability for OSI and TAI. The linear $\kappa_{\mathrm{a}}$-dependence of $\Delta T^{\mathrm{c}}$ for OSI is too weak in order to be shown in figure 1 .

The dotted line is given by $\kappa_{\mathrm{a}} k^{2}=\zeta \hat{\chi}^{2} ;$ its distance to the $\Delta T^{\mathrm{c}}$-axis is proportional to $H^{2}$. Thus, as in the case of nematics [6] the intersection of the boundary lines of the regions to OSI and TAI respectively, changes with the magnitude of the external magnetic field.

3. Mechanism. - For the Bénard instability in simple fluids the interplay of heat conduction and shear viscosity terms with the buoyancy force leads to a coupling of the variations of temperature and velocity i.e. of $\delta T$ and $V_{z}$ and vice-versa [15].

In smectics $\mathrm{A}$ and cholesterics, however, there exists an additional coupling between $\delta T$ and $V_{z}$ via the variations of the additional degree of freedom $\delta R_{z}$. This additional coupling causes the system to become overstable at threshold, i.e. oscillations with $\omega_{c}$ occur.

Mathematically speaking this difference between the Bénard instability in simple fluids and the OSI in smectics A and cholesterics arises from the fact that the differential operator associated with the system of differential equations for linear deviations from the heat conduction state is self adjoint in the Bénard case whereas it is nonhermitian in the OSI.

According to equations (1) a variation $\delta R \sim \cos \omega t$ generates variations $V_{z}$ and $\delta T$ which are partially out of phase $\left(\sim \cos \left(\omega t+\Phi_{i}\right)\right)$. This time delayed answer of the system leads to an undamped oscillatory behaviour at threshold.

In the limit $\hat{\mu}^{4} k^{-2}, \omega \gg \hat{\kappa}^{2}, \zeta \hat{\chi}^{2} / \delta R_{z}$ and $\delta T$ are in phase whereas $V_{z}$ is out of phase by 90 degrees. This behaviour is different from that of nematics in the planar configuration whose temperature variations are in phase with the velocity field whereas the direction variations have a 90 degree phase delay with respect to the velocity field.

In figure 2 we have plotted schematically the space time pattern for the variations $\delta R_{z}, \delta T$ and $V_{z}$ in the

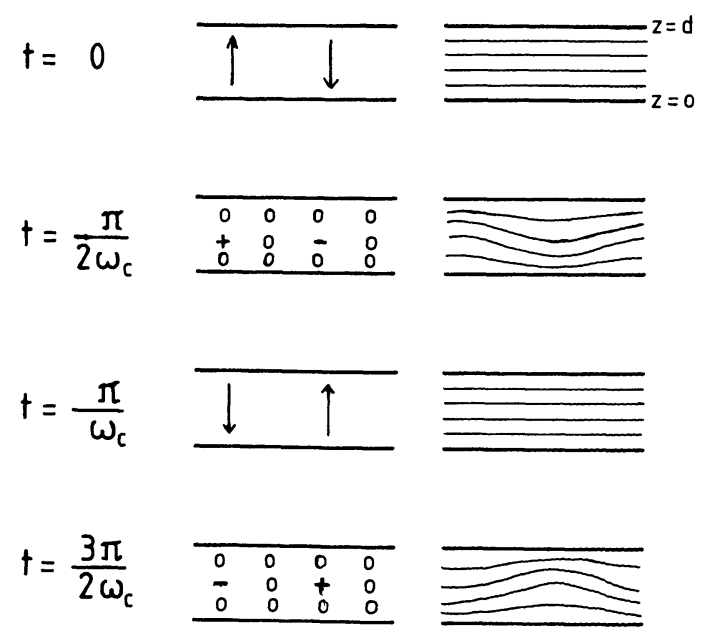

Fig. 2. - Mechanism of the oscillatory instability in a smectıc A liquid crystal $: \uparrow$ hydrodynamic velocity $V_{z},+$ warmer, - colder regions. 
above mentioned limit. There is no permeation. The elastic oscillations of the layers are driven by the external temperature gradient.

In conclusion our analysis shows that an oscillatory convective instability can occur in smectics $A$ and cholesterics. We hope that our theoretical prediction will stimulate experimental research like in the case of nematics where the oscillatory instability was also predicted theoretically [9] and has been found afterwards experimentally $[5,6]$.

\section{References}

[1] Graham, R., Phys. Rev. Lett. 31 (1973) 1479.

[2] Graham, R., Phys. Rev. A 10 (1974) 1762.

[3] Pieranski, P., Dubois-Violette, E. and Guyon, E., Phys. Rev. Lett. 30 (1973) 736.

[4] Dubois-Violette, E., Guyon, E., Pieranski, P., Mol. Cryst. Liq. Cryst. 26 (1974) 193.

[5] Guyon, E., Pieranski, P., Salan, J., C.R. Hebd. Séan. Acad. Sci. Paris 287B (1978) 41.

[6] Guyon, E., Pieranski, P., Salan, J., J. Fluid Mech. 93 (1979) 65.

[7] Dubois-Violette, E., C.R. Hebd. Séan. Acad. Sci. Paris 273B (1971) 923.
[8] Dubois-Violette, E., Solid State Commun. 14 (1974) 767.

[9] LeK Kerkerker, H. N. W., J. Physique Lett. 38 (1977) L-277.

[10] Dubois-Violette, E., J. Physique 34 (1973) 107.

[11] Parsons, J. D., J. Physique 36 (1975) 1363.

[12] Pleiner, H. and Brand, H., to be published.

[13] Brand, H. and Pleiner, H., J. Physique 41 (1980) 553.

[14] Martin, P. C., Parodi, O. and Pershan, P. S., Phys. Rev. A 6 (1972) 2401.

[15] Chandrasekhar, S., Hydrodynamic and Hydromagnetic Stability (Clarendon, Oxford) 1961. 